\title{
OPTIMISATION OF THE DESIGN OF UAV WING
}

\author{
Alexander $\mathrm{J}^{1}$., Prakash $\mathrm{S}^{2}$., Augustine $\mathrm{BSM}^{3}$ \\ ${ }^{1,2,3}$ Department of Aeronautical Engineering, Sathyabama University \\ Email: 1 vsjalexander@ rediffmail.com
}

\section{Abstract}

This paper presents the research extensively carried out based on the static analysis for finding the optimum design of wing structure. The two types of wings (Rectangular wings) for unmanned airplanes were chosen and designed at the basis of $M=0.21$ and at $M=0.4$ respectively. Further it also covers both the aerodynamic and structural designs. The aerodynamics study was achieved by using the vortex lattice method using XFLR-CFD software and the structural analysis was achieved by using the CATIA V5 package consisting of isotropic and composite materials. A wing structure consists of spars, ribs, reinforcements and skin and they were optimized considering two aspects of weight minimization, and critical load maximization. The wing carries an elliptically distributed load along the span. The design variables are based on the positioning of spars and ribs of the structure. The out come of the research clearly indicates the significant results in terms of objective functions obtained through the optimization procedures. When composite material is used instead of isotropic material mass saving of $34 \%$ is achieved. The optimum design for each wing was obtained according to the mass, stress and displacement. This could be seen in the presentation subsequent

Keyworks: Structural analysis, optimization procedures.

\section{Nomenclature}

Vn: Normal velocity, $(\mathrm{m} / \mathrm{s})$

$\mathrm{V} \infty$ : free stream velocity, $(\mathrm{m} / \mathrm{s})$

$\Theta$ Flight path angle, (deg.)

$W_{i}$. Induced flow component, $(\mathrm{m} / \mathrm{s})$

$\alpha$ : Angle of attack, (deg.)

$A_{i j:}$ Influence coefficient

$L_{i}$ Lift of panel $i,(\mathrm{~N})$

$\Gamma$ : Strength of vortex $\left(\mathrm{m}^{2} / \mathrm{s}\right)$

$L$ : Lift of wing, $(\mathrm{N})$

$D_{i}$. Drag of panel $i,(\mathrm{~N})$

D: Drag of wing, $(\mathrm{N})$

CLcom: Lift coefficient in compressible flow

CLincom: Lift coefficient in incompressible flow

M: Mach number

E: Young s modulus, (pa)

G: Shear modulus, (pa)

$v$ : Poisson $s$ ratio

$\rho:$ Density, $\left(\mathrm{kg} / \mathrm{m}^{3}\right)$

D.O.F.: Degree of freedom

M.A.C.: Mean aerodynamic chord (m)
A/C: Aircraft

V.M.: Von-Mises (Mpa)

Gr/Ep: Graphite/epoxy

H.M Gr/Ep: High modulus graphite/epoxy

Sg/Ep: Glass/epoxy

Wto - total aircraft weight

Ww :weight of wing structure

Wf :weight of fuel stored in wing

$L$ :length of wing

Lf :length of fuel tank within wing

Co :chord length at wing root

$\mathrm{Ct}$ :chord length at wing tip

Co :width of fuel tank at wing root

Ctf width of fuel tank at $L f$

\section{INTRODUCTION}

An Unmanned Air Vehicle (UAV) is an unpiloted aircraft. UAV are becoming standard means of collecting information. The optimum structural design of an $\mathrm{A} / \mathrm{C}$ wing is an important factor in the performance of the airplanes i.e. obtaining a wing with a high stiffness/weight ratio and sustaining the unexpected loading such as gust and maneuvering situations. This is accomplished by studying the different design parameters required to specify the wing geometry. The 
idea of the structural optimization in the classical sense, has been considered to be the minimization of structural mass by varying member sizes or shell thickness of a model in which the geometry remains unchanged. Therefore many studies have been made during the last years to find the structural optimization.

One of the major loading is aerodynamic loading. Aerodynamic characteristics of the UAV[7] vary with certain parameters like the angle of attack and others. Experimental works on UAVs have been conducted in many places with various aerofoil profiles but enough work with the Computational Fluid Dynamics (CFD) analysis is not available that much till now. The present work contains mainly CFD[8] analysis to determine the flow pattern and the aerodynamic characteristics of an UAV. The shape of an aircraft is designed to make the airflow through the surface to produce a lifting force in most efficient manner. In addition to the lift, a force directly opposing the motion of the wing through the air is always present, which is called a drag force. The angle between the relative wind and the chord line is the angle of attack of the aerofoil. The lift and drag forces developed by an aircraft will vary with the change of angle of attack. The cross sectional shape obtained by the intersection of the wing with the perpendicular plane is called an aerofoil. Here NACA $0012^{(10)}$ symmetric aerofoil profiles have been used for the present research work. The lift force increases almost linearly with the angle of attack until a maximum value is reached where upon the wing is said to stall.

The shape of the drag force vs. angle of attack is approximately parabolic. It is desirable for the wing to have the maximum lift and smallest possible drag.

To develop a more accurate model[2] for such optimal design studies, the Structure of the wing that combines the composite[11] and isotropic materials and compare the wing with the wing that design only from isotropic material in order to obtain high strength/weight ratio for finding optimum design.

Types of load acting on the aircraft wing

1. Aerodynamic loading

2. Structural loading

3. Fuel Weight

The optimization chain consists of:
1. Aerodynamic grid generation

2. CFD Analysis by using XFLR software

3. Parametric CFD model generation

4. Structural grid generation

5. FEM Analysis and structure sizing

\section{AIM AND SCOPE OF THE PRESENT INVESTIGATION}

A light rectangular wing of an UAV with NACA0012 airfoil is developed using CATIA software and the static analysis of the wing by CATIA V5 package has been presented and used to determine the optimum design for the wing. This operation involves using the vortex lattice method to obtain the aerodynamic load(lift) for rectangle wing. This aerodynamic result (lift) is combined[4] with the fuel weight and structural weight of the wing, and it is used to simulate the wing loading on the wings during the static analysis.

Some of the parameters of aerofoil and properties of air have been kept constant and some have been varied. The flow of air over the aerofoil is varied as per requirement. The chord length of the aerofoil is $450 \mathrm{~mm}$. (10)The free stream airflow has been kept 60 $\mathrm{m} / \mathrm{s}$ and the effect of the temperature in the study has been neglected. The density of air $\left(\rho_{0}\right)=1.22 \mathrm{~kg} / \mathrm{m}^{3}$, operating pressure $\left(\rho_{0}\right)=0.101 \mathrm{MPa}(1.01 \mathrm{bar})$ and absolute viscosity $(\mu)=1.789 \times 10^{-5} \mathrm{~kg} / \mathrm{m}-\mathrm{s}$. The Reynolds Number has been considered as variable.

\section{METHOD OF ANALYSIS AERODYNAMICS}

The aircraft motion through the atmosphere produces forces called the aerodynamic forces mainly the lift and drag forces and the wing is the major source of this aerodynamic force. The wing is that surface of aircraft which supports the aircraft by means of the dynamic reaction on the air producing pressure distribution. This pressure distribution on the wing changes with different wing angles of attack and flight condition.

Methods of calculating aerodynamic load (Lift)

1. Prandtl's classical lifting line theory

2. Vortex lattice numerical method

3. Panel method 
In this research work vortex lattice method is used

Vortex lattice method[8]

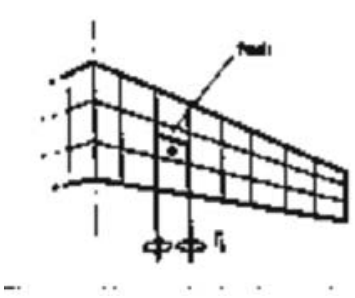

Fig. 1. Wing panels

The whole wing is divided into many numbers of panels and each panel is considered as a bound vortex. The required strength of the bound vortex on each panel is to be calculated by applying a surface flow boundary condition. The equation used is the usual condition of zero flow normal to the surface. For each panel the condition is applied at the $3 / 4$ chord position along the centre line of the panel[9]. The normal velocity is made up of a free stream component and an induced flow component, as shown in Fig. (2)

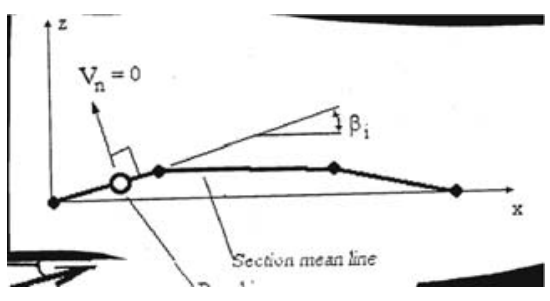

$\alpha \quad \mathrm{V}$

Panel $i$

Fig. 2. Velocity component of the panel $i$ on the wing.

$$
V_{n}=0=\sin \theta+W_{i}
$$

The induced component is a function of strength of all vortex panels on the wing. Assuming small angles

$$
\begin{array}{ll}
\sin \theta=\sin (\alpha-\beta)=(\alpha=\beta)=-d z / d x & \ldots \text { [2] } \\
W_{i}=\sum_{i=1}^{n} A_{i j} \Gamma_{j} & \ldots \text { [3] } \\
\sum_{i=1}^{n} A_{i j} \Gamma_{j}=-V \sin (\theta) & \ldots \text { [4] }
\end{array}
$$

Where $\theta$ is flight path angle

$A_{i j}$ is the influence coefficient which represents the induced flow on panel $/$ due to the vortex on panel $j$

A solution for the strength of the vortex lines on each panel is found by solving the matrix of equations the lift coefficient for the wing at a given angle of attack will be obtained by integrating the panel lift distribution. The lift on a particular panel can be found using the Kutta law.

$$
\begin{aligned}
& L_{i}=\rho V_{\infty} \Gamma_{j} 2 \mathrm{~K} \text { Lift of panel } i \\
& L=\sum_{i=1}^{n} L_{i} \text { Lift of wing }
\end{aligned}
$$

The down wash velocity induced on a panel can be calculated once the strength of the wing loading is known. The variation between local flow angles of the panel and the free stream velocity can be found. consequence of this down wash flow is that the direction of action of each panels lift vector is rotated relative to the free stream direction. The local lift vectors are rotated backward and hence give rise to a lift induced drag. By integrating the component of panel lift coefficient that acts parallel to the free stream across the span then the induced drag coefficient can be found.

$$
\begin{aligned}
& D_{i}=\rho V_{\infty} i \Gamma 2 K \text { Drag of panel } i \\
& D=\Sigma_{i=1}^{n} D_{i} \text { Drag of the wing }
\end{aligned}
$$

The induced flow angle $(\alpha i)$ represents the amount of rotation of the lift vector backward and must be calculated from the velocities induced on the bound vortex of the panel by other panels and the free stream.

Pitching moment about the wing root loading edge can be calculated by summing the panel lift multiplied by a moment arm which extends in the $x$-direction from the loading edge of the wing to the centre of the bound vortex for the panel.

\section{ANALYTICAL MODELING OF AIRCRAFT WING LOADS}

\section{A. Lift}

We assume an elliptical distribution for lift across the length of the wing, resulting in the following expression forlift profile[4]

$$
q^{1}(X)=k a \sqrt{L^{2}-X^{2}}
$$

where $L=$ length of wing

$$
x=\text { position along wing }
$$

$k a=$ lift profile coefficient 


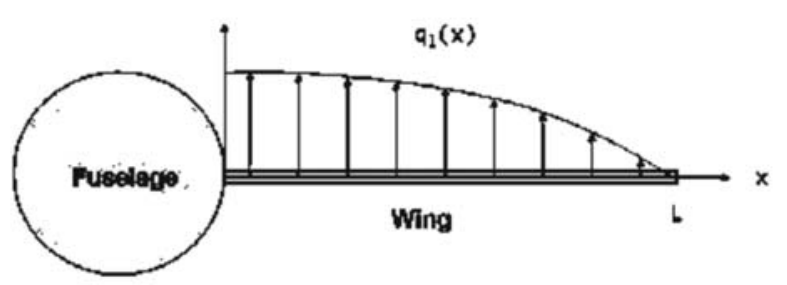

Fig. 3. Derivation of Lift

We can determine the total lift by integrating across the length of the wing:

Within the notebook interface we define $q l(x)$ and calculate its integral (Figure 5). We incorporate math equations, descriptive text, and images into our calculations to clearly document our work.

Through integration, we find that

$$
\text { Lift }=\pi L^{2} k a / 4
$$

We determine ka by equating the lift expression that we just calculated with the lift expressed in terms of the aircraft's load factor. In aircraft design, load factor is the ratio of lift to total aircraft weight:

$$
n=\mathrm{Lift} / \mathrm{Wt}
$$

Load factor equals 1 during straight and level flight, and is greater than 1 when an aircraft is climbing or during other maneuvers where lift exceeds aircraft weight.

Equating two lift expressions,

$$
W t_{0} n / 2=\pi L^{2} \mathrm{ka} / 4
$$

and solve for the unknown ka term. Our analysis assumes that lift forces are concentrated on the two wings of the aircraft, which is why the left-hand side of the equation is divided by 2 . We do not consider lift on the fuselage or other surfaces

$$
\begin{aligned}
& q^{1}(X)=\underline{k a \sqrt{L^{2}-X^{2}}} \\
& \text { Lift }=\int k a \sqrt{L^{2}-X^{2}} d X
\end{aligned}
$$

Plugging ka into our original $q l(x)$ expression, we obtain an expression for lift:

An analytical model like this helps us understand how various parameters affect lift. For example, we see that lift is directly proportional to load factor $(n)$ and that for a load factor of 1 , the maximum lift , occurs at the wing root $(x=0)$

\section{B. Weight of Wing Structure}

We assume that the load caused by the weight of the wing structure is proportional to chord length (the width of the wing), which is highest at the wing base $\left(C_{0}\right)$ and tapers off toward the wing tip $\left(C_{t}\right)$. Therefore, the load profile can be expressed as. We define qw $(X)$ and integrate it across the length of the wing to calculate the total load from the wing structure:

We then equate this structural load equation with the structural load expressed in terms of load factor and weight of the wing structure (Wws) and solve for $k w$.

Plugging $k w$ into our original $q_{w}(x)$ expression, we obtain an analytical expression for load due to weight of the wing structure.

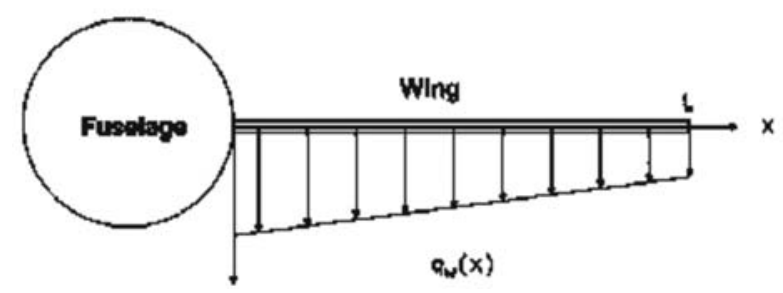

Fig. 4. showing the weight of wing structure

$$
\begin{aligned}
& q_{w}(x)=-\frac{W w s n\left(C_{0}-\frac{x}{L\left(C_{0}+\right.}\right.}{\left[\frac{W w s}{2}=\frac{L k w\left(C_{0}+C_{t}\right)}{2}\right.} \\
& {[\mathrm{kw}:=- \text { solve }(\text { eqn, kw) }} \\
& \left\{-\frac{W w s n}{L\left(C_{0}+C_{t}\right)}\right\}
\end{aligned}
$$

\section{Weight of Fuel Stored in Wing}

We define the load from the weight of the fuel stored in the wing as a piecewise function where load is zero when $x>L f$. We assume that this load is proportional to the width of the fuel tank, which is at its maximum at the base of the wing (Cof) and tapers off as we approach the tip of the fuel storage tank 
(Ctf). We derive $q f(x)$ in the same way that we derived $q w(x)$, resulting in an equation of the same form:

$$
q f(x)=\left\{\begin{array}{cc}
0 & \text { if } \mathrm{Lf}<\mathrm{x} \\
-\frac{w f n\left(C_{o f} \frac{x\left(C_{o f}-C_{t f}\right.}{L f}\right)}{L f(C o f+C t f)} & \text { if } x \leq \mathrm{Lf} .
\end{array} .\right.
$$

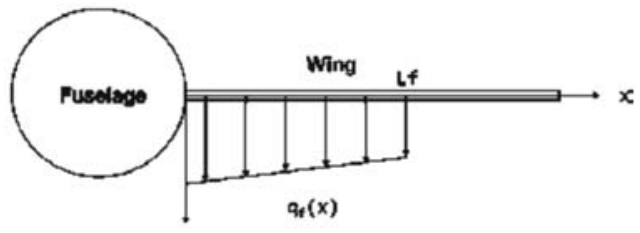

Fig. 5. showing the weight of fuel

\section{Total Load}

We calculate total load by adding the three individual load components. This analytical model gives a clear view of how aircraft weight and geometry parameters affect total load.

$$
q f(X)= \begin{cases}\frac{\pi\left(2 \operatorname{Co} W t o \sqrt{L^{2}-X^{2}}+2 \operatorname{Ct} W t o \sqrt{L^{2}-X^{2}}-X \operatorname{Co} L W w s+X \operatorname{Co} W w s X-X \operatorname{Ct} W w s X\right)}{L^{2} X(C o+C t)} & \text { if } L f<x \\ \frac{2 W t o n \sqrt{L^{2}-X^{2}}}{L^{2} X}-\frac{W w s n(C t X-\operatorname{Co} X-\operatorname{Co} L)}{L^{2}(C o+C t)}-\frac{W f n(C t f X-\operatorname{Cof} X+\operatorname{Cof} L f)}{L t^{2}(\operatorname{Cof}+C t f)} & \text { if } x \leq L f\end{cases}
$$

\section{MODELING AND ANALYSIS}

Aerodynamic model is developed and analyzed by using CFD-XFLR software and the structural model is generated by CATIA V5 and is analysed by using ansys.

\section{A. CFD ANALYSIS}

The wing is designed with single aerofoil profile. The aerofoil number is NACA 0012 which is a symmetric aerofoil.

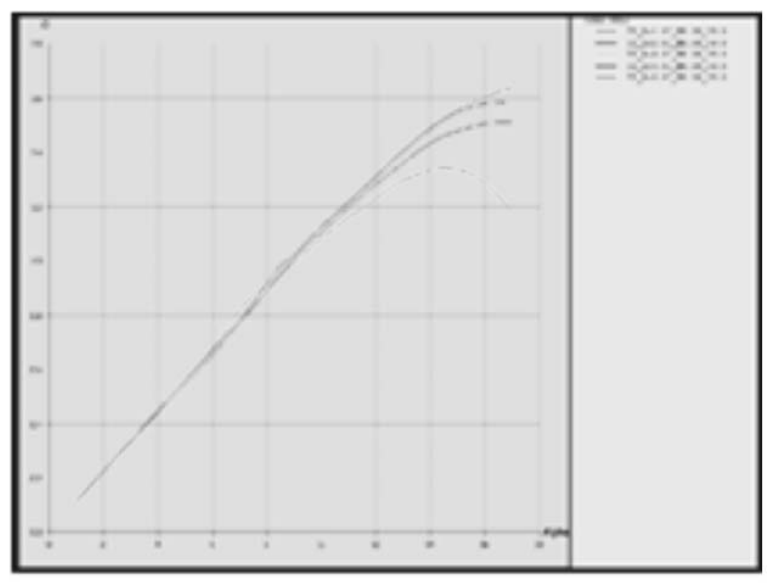

Fig. 6. Cl Vs Alpha Curve At various Reynolds number

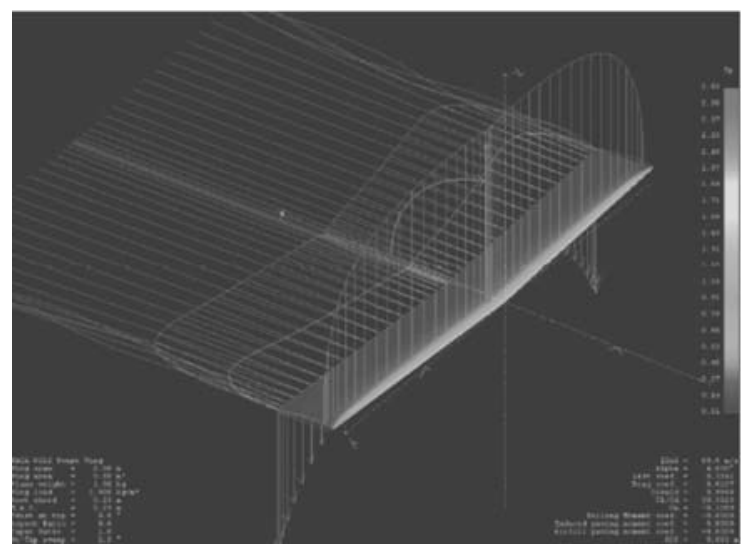

Fig. 7. Lift Distribution over the wing

Table 1. Main characteristics of the wings

\begin{tabular}{|l|c|}
\hline \multicolumn{1}{|c|}{ Characteristic } & Rectangle wing \\
\hline Wing span (m) & 3.35 \\
\hline Gross area (m2) & 1.53 \\
\hline Aspect ratio & 7.33 \\
\hline Taper ratio & 1 \\
\hline Tip chord (m) & 0.45 \\
\hline Root chord (m) & 0.45 \\
\hline M. A. C. (m) & 0.45 \\
\hline Sweep angle of leading edge (deg.) & 0 \\
\hline Section profile & NACA 0012 \\
\hline
\end{tabular}


Results of the theoretical aerodynamic analysis by CFD-XFLR. Table (1) shows the main characteristics of the wings. The aerodynamic results (lift) are used to simulate the wing loading on the wings during the static stress analysis.

Table 2. Calculated polar through CFD analysis XFLR5_v4.17 polar for: NACA 0012 Mach $=0.2 \mathrm{Re}=1.000$ e 6

\begin{tabular}{|c|c|c|c|}
\hline AOA & Cl & Cd & Cm \\
\hline 1 & 0.11 & 0.0051 & 0.001 \\
\hline 3 & 0.32 & 0.0058 & 0.0022 \\
\hline 5 & 0.54 & 0.0078 & 0.0054 \\
\hline 7 & 0.82 & 0.01 & 0.0084 \\
\hline 9 & 0.992 & 0.013 & -0.0084 \\
\hline 11 & 1.15 & 0.0177 & 0.0012 \\
\hline 13 & 1.29 & 0.023 & 0.0102 \\
\hline 15 & 1.33 & 0.035 & 0.021 \\
\hline 17 & 1.1801 & 0.086 & 0.0093 \\
\hline
\end{tabular}

The aerodynamic results are taken for a range of wing incidences Figs.(7) and and only the maximum wing loadings are applied to the wings to serve wing stress conditions

Table 3. Load acting at various portion of the wing

\begin{tabular}{|c|c|}
\hline distance (m) & load N \\
\hline 0.25 & 73.39 \\
\hline 0.5 & 36.352 \\
\hline 0.75 & 17.885 \\
\hline 1 & 17.471 \\
\hline 1.5 & 16.235 \\
\hline 2 & 14.349 \\
\hline 2.5 & 4.59 \\
\hline 3 & -0.594 \\
\hline 3.35 & -8.763 \\
\hline
\end{tabular}

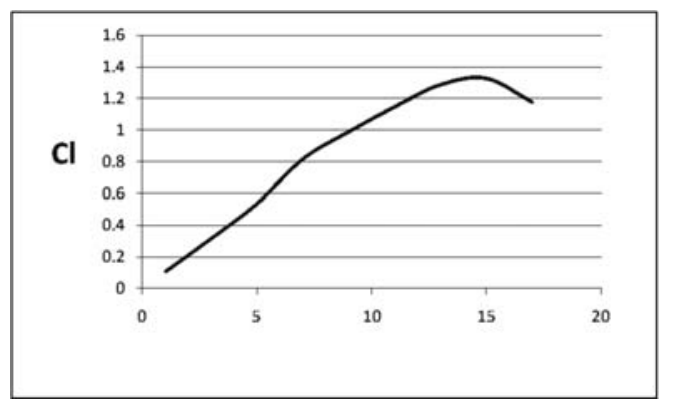

Fig. 8. Alpha Vs $\mathrm{Cl}$ Curves at Mach no 0.2

\section{CALCULATION OF WING LOADING}

Plot of individual load components and total load along the length of the wing.

We see that lift is the largest contributor to total load and that the maximum load occurs at the end of the fuel tank. Fuel load also contributes significantly to the total load, while the weight of the wing is the smallest contributor.

While it is useful to visualize wing loads, what really concerns us are the shear force and bending moments resulting from these loads. We need to determine whether worst- case bending moments experienced by the wing are within design limits

$$
\begin{aligned}
W t o & =108 \mathrm{~kg} \\
W W & =14.17 \mathrm{~kg} \\
W f & =32 \mathrm{~kg} \\
L & =3.35 \mathrm{~m} \\
L f & =2.3 \mathrm{~m} \\
C o & =0.45 \mathrm{~m} \\
C t & =0.45 \mathrm{~m} \\
C o & =0.275 \mathrm{~m} \\
C t f & =0.275 \mathrm{~m}
\end{aligned}
$$

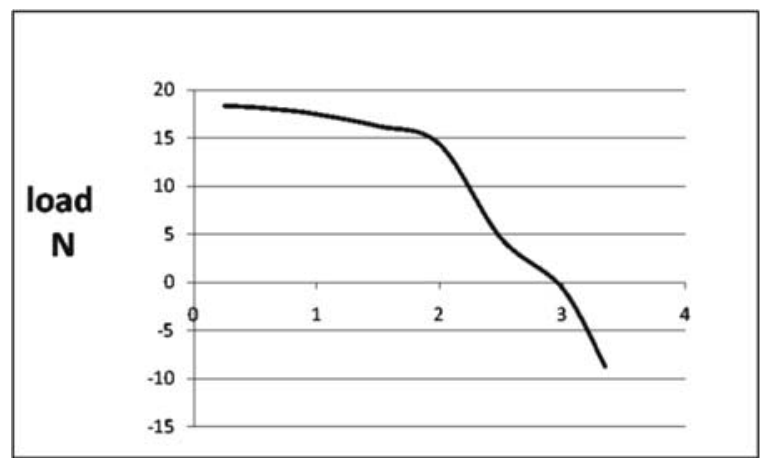

Fig. 9. Load distribution over the wing 


\section{STRUCTURAL STATIC ANALYSIS}

Structural analysis is probably the most common application of the finite element method. The static analysis is used to determine displacements, stresses, etc. under static loading conditions. In this work the structural static analysis was achieved by using the CATIA V5 package in order to obtain stress and displacement distribution in the wings by using isotropic and composite material which are used commonly these days especially for unmanned A/C. The CATIA V5 program has many finite element analysis capabilities, ranging from a simple, linear, static analysis to a complex, non linear, transient dynamic analysis. A typical CATIA V5 analysis has following distinct steps:

(i) Build the model.

(ii) Apply loads

(iii) Obtain the solution.

(iv) Review the results.

\section{A. Materials}

Historically, aluminum materials have been the primary material for aircraft and space craft construction. Today, structural weight and stiffness requirements have exceeded the capability of conventional aluminum, and high-performance payloads have demanded extreme thermo-elastic stability in the aircraft design environment. During the past decades, advanced composite materials have been increasingly accepted for aircraft and aerospace structural materials by numerous developments and flight applications. Composite materials are those containing more than one bonded material, each with different structural properties.

For the sake of simplicity two types of materials have been used for the optimization procedure.One is isotropic material that wing configuration is completely made up of Aluminum or titanium (7075-T6, adv. Aluminum, Ti6A 14V). Another one is composite material that wing configuration is Graphite/Epoxy or S-glass/Epoxy.The material properties are shown in Table (4)
Table 4. Material properties ${ }^{(12)}$

\begin{tabular}{|c|c|c|}
\hline Material properties & $\begin{array}{c}\text { Aluminum } \\
\text { allay 7075 -T6 }\end{array}$ & $\begin{array}{c}\text { Graphite/ } \\
\text { Epoxy }\end{array}$ \\
\hline V.M. stress (MPa) & 103 & 109 \\
\hline UY(m) & 0.026 & 0.027 \\
\hline Stress ratio \% & 21.91 & 23.19 \\
\hline Mass (Kg) & 5.58 & 2.01 \\
\hline Yield/weight * $10^{6}\left(1 / \mathrm{m}^{2)}\right.$ & 5.96 & 7.76 \\
\hline Weight/Area $\left(\mathrm{N} / \mathrm{m}^{2}\right)$ & 51.48 & 39.56 \\
\hline
\end{tabular}

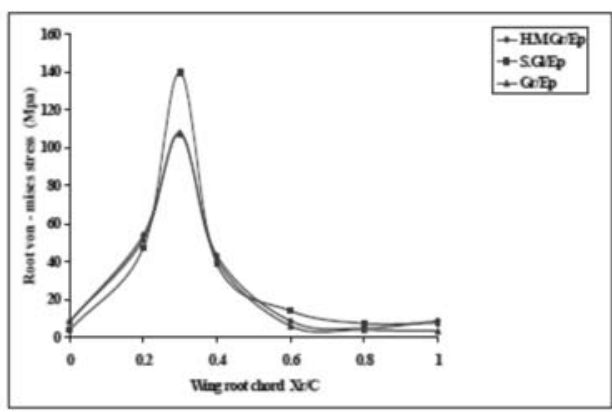

Fig. 10. Catia Wing Model

\section{B. Optimization Procedure}

The wing structural design problem is composed into two levels in a hierarchical structure at the first level, the wing configuration is completely made up of isotropic material and the following design parameters were investigated such as number of stiffeners, skin thickness, cross sectional area of stiffener and the type of material. The second level wing substructure (spars, stiffener) is made of isotropic material and the skin panel is made of composite material. Various types of composite materials are also considered. Then from the results we take the optimum design.

Table 5. Variation of von misses stress due to variation of no of ribs

\begin{tabular}{|c|c|c|c|}
\hline No. of ribs properties & 3 & 4 & 5 \\
\hline V.M. stress $(\mathrm{M} \mathrm{Pa})$ & 129 & 129 & 130 \\
\hline UY $(\mathrm{mm})$ & 0.022 & 0.022 & 0.022 \\
\hline Stress ratio \% & 29.57 & 29.57 & 30.00 \\
\hline Mass $(\mathrm{Kg})$ & 1.991 & 2.01 & 2.11 \\
\hline Yield/weight ${ }^{*} 10^{6}\left(\mathrm{~N} / \mathrm{m}^{2}\right)$ & 8.02 & 7.86 & 7.72 \\
\hline Weight/Area $\left(\mathrm{N} / \mathrm{m}^{2}\right)$ & 38.27 & 38.98 & 39.75 \\
\hline
\end{tabular}




\section{RESULTS AND DISCUSSIONS}

The structural analysis was achieved by using the CATIA V5 package in order to obtain stress and displacement distributions in the wings by using isotropic and composite material to find the optimum design. The work involves the investigation effects of changing the skin shell thickness $(0.001-0.003 \mathrm{~m})$, the stiffener beam area $\left\{\left(44 \mathrm{~mm}^{2}-81 \mathrm{~mm}^{2}\right)\right.$, adding ribs span wisely $(3-5)$ adding stringers chord wisely $(0-5)$. The optimum design parameter was achieved, where the skin thickness $(0.001 \mathrm{~m})$ for rectangle wing and the stiffener $(3 \times 5)$. The optimum isotropic material is (7075-T6) . Also the optimum cross section area of stiffener equal to $\left(44 \mathrm{~mm}^{2}\right)$

Table (5) shows the Von-Mises stress and (Uy) results for rectangle wing when adding the ribs span wisely, increasing the number of ribs span wisely gives small reduction effect on the Von-Mises stress and displacement distribution.

Table 6.(a) Result of isotropic materials

\begin{tabular}{|c|c|c|c|}
\hline $\begin{array}{c}\text { Isotropic } \\
\text { material }\end{array}$ & $7075-\mathbf{T 6}$ & $\begin{array}{c}\text { Adv } \\
\text { aluminium }\end{array}$ & Ti6V 14V \\
\hline$E\left(\mathrm{~N} / \mathrm{m}^{2}\right)$ & $71 E+9$ & $S 2.7 E+9$ & $11032 E+9$ \\
\hline $\mathrm{V}$ & 0.33 & 0.318 & 0.29 \\
\hline $\begin{array}{c}\mathrm{YaH} i \text { trees } \\
\left(\mathrm{N} / \mathrm{m}^{2}\right)\end{array}$ & $+70 E+6$ & $+2+E+6$ & $190 E+4$ \\
\hline$\rho\left(\mathrm{Kp} / \mathrm{m}^{2}\right)$ & 1800 & 29064 & 4429 \\
\hline
\end{tabular}

Table 6.(b) Result of composite materials

\begin{tabular}{|c|c|c|c|}
\hline Composite material & HM Gr/Ep & Gr/Ep & Sp/Hp \\
\hline$E 1$ (Gpa) & 1379 & $1+5$ & 55 \\
\hline$E 2=E 3$ Gpa & $1+18$ & 10 & 14 \\
\hline$V 12=23=13$ & 0.21 & 0.25 & 0.28 \\
\hline$G 12=G 23=G 13$ (Gpa) & 184 & 48 & 74 \\
\hline$\rho(K p / m)$ & 1743.84 & 1380 & $1593+2$ \\
\hline
\end{tabular}

Table (6) shows comparison of the results between the isotropic and composite material. The goal comparison is to emphasize that the wing total mass is reduced and Von-Mises stress increases when composite material is used instead of isotropic material.
Figures(12), (13) show the Von-Mises stress distribution in case of using isotropic and composite materials for rectangular wing, in both cases the root Von-Mises variation increases through out the $(0.2-0.35)$ chord zone.y

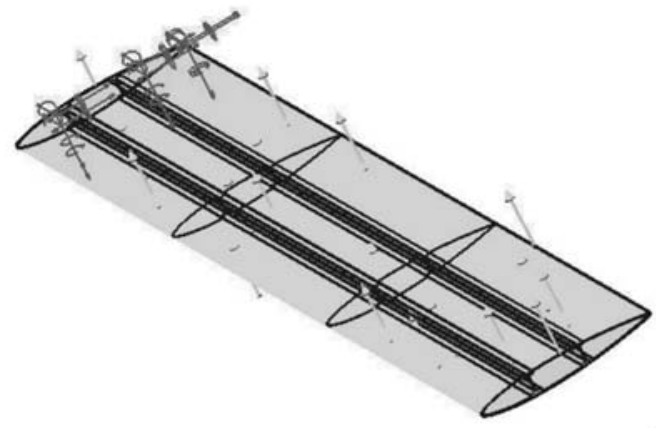

Fig. 11. Effect of von mises stress in changing the composite material

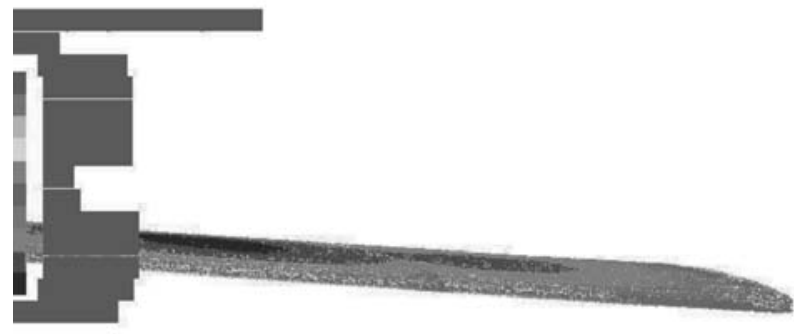

Fig. 12. Contours of Von misses stress $(\mathrm{Pa})$ distribution for isotropic wing

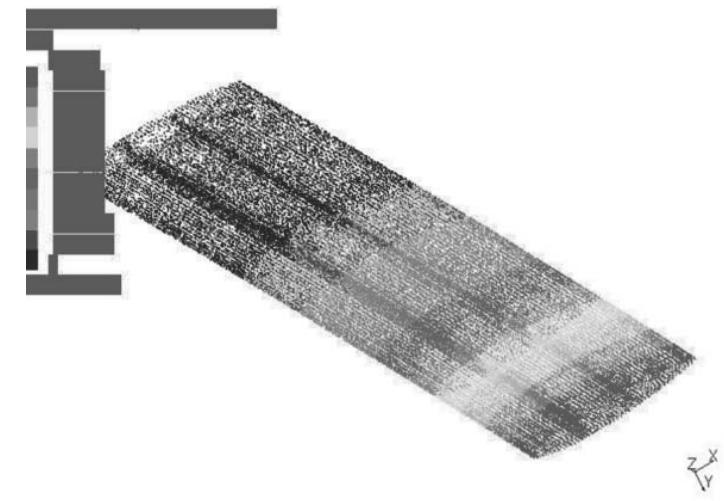

Fig. 13. Contours of Von misses stress $(\mathrm{Pa})$ distribution for composite wing

Figures shows the displacement distribution of Isotropic and Composite wings 


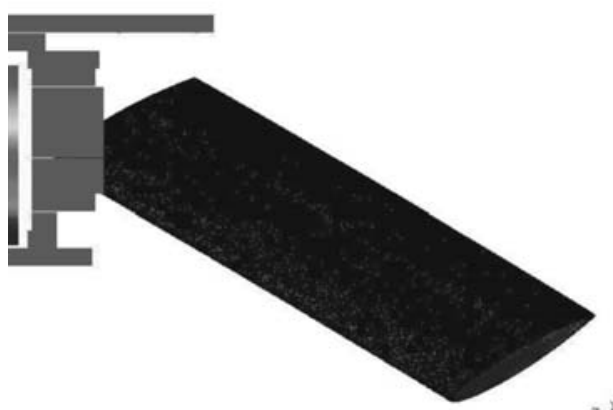

Fig. 14. Contours of Displacement distribution of isotropic wing

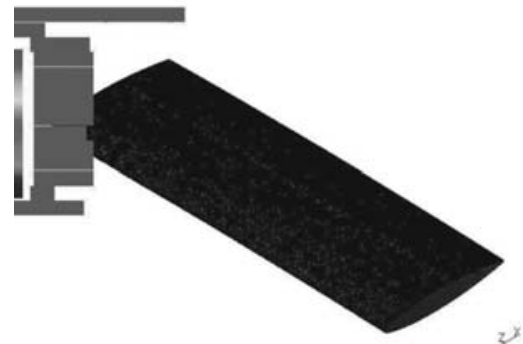

Fig. 15. Contours of Displacement distribution of Composite wing

\section{SUMMARY AND CONCLUSSION}

The static analysis of the wing by CATIA V5 package has been presented and used to determine the optimum design for the wing. This operation involves using the vortex lattice method to obtain the aerodynamic result at $(M=0.2)$ for rectangle wing. This aerodynamic result (lift) is used to simulate the wing loading on the wings during the static analysis and also using isotropic and composite materials in design the parts of wing. Therefore the major and general observations and conclusions for this work can be listed below:

1. From the Aerodynamic results it can be noticed that lift coefficient increases with the increase of Mach number at the same angle of attacks due to the compressibility.

2. From the structural results it is noticed that increasing the skin thickness is considered as an important factor in reducing the stress and displacement levels compared to the increase of, cross section area of beam and number of layers.

3. Increasing the ribs span wisely does not change noticeable the Von-Misses stress distribution.
4. Also it is found that the mass saves (34\%) when composite material is used instead of isotropic material wing.

5. It is desirable to use composite materials in design of the skin of a wing

\section{REFERENCES}

[1] lyengar N.G.R., and Joshi S.P., 1986 "Optimal Design of Antisymmetric Laminated Composite Plates", Journal of Aircraft, Vol. 23, No. 5,

[2] Confield R.A., Granhi R.V., and Venkayya V.B., 1988, "Optimum Design of Structures with Multiple Constraints", AIAA Journal, Vol. 26, No.1 p.78.

[3] Tulaburkara E.G., Venkatraman A., Ganesh V., 2007 An example of preliminary design of Jet plane

[4] Dan Doharty, Analytical modeling of aircraft wing using matlab, Matlab digest

[5] Thomas Chamberlain, Methods of calculating aerodynamic load on aircraft structures

[6] Tobias F.Wunderlich 2008." Multidisciplinary wing design and optimization for transport aircraft" DRL Institute of aeronautics and flow Technology

[7] Shawn E.Gano,John E.Renaud 2002 " Optimized unmanned aerial vehicle with wing Morphing", , AIAA Journal ".

[8] 1998 "Aerodynamics of 3-D lifting surfaces using vortex lattice method" NASA Journal

[9] Bertin J.J., and Smith M.S., 2000 "Aerdynamics for Engineering Students", Department of Aeronautical Engineering, University of Sydney.

[10] Alimul Rajib, Bhuiyan Shameem Mahmud Ebna Hai and Md Abdus Salam, Department of Mechanical Engineering, Military Institute of Science and Technology, Dhaka-1216, Bangladesh."Design analysis of UAV using NACA 0012 Airfoil'.

[11] Kao A., Mechanics of composite materials.

[12] Rol P.J., Marris D.N., and Schrage D.P.,1995 "Combined Aerodynamic and Structural Optimization of a High-Speed Civil Transport Wing", School of Aerospace Engineering, American Institute of Aeronautic and Astronautic, Inc.,

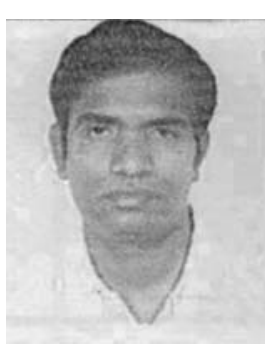

J.Alexander is an Assistant professor at the Department of Aeronautical Engineering in Sathyabama University. His area of interest include Aerodynamics and Structural analysis of UAV, Composite structures 